\title{
Apparent close approaches between near-Earth asteroids and quasars
}

\section{Precise astrometry and frame linking ${ }^{\star}$}

\author{
D. A. Nedelcu ${ }^{1,2}$, M. Birlan ${ }^{1}$, J. Souchay ${ }^{3}$, M. Assafin ${ }^{4}$, A. H. Andrei ${ }^{4,5}$, O. Bădescu ${ }^{2}$, P. Popescu ${ }^{2}$, and P. Paraschiv ${ }^{2}$ \\ 1 Institut de Mécanique Céleste et de Calcul des Éphémérides (IMCCE), Observatoire de Paris, 77 avenue Denfert-Rochereau, \\ 75014 Paris Cedex, France \\ e-mail: [nedelcu;mirel.birlan]@imcce.fr \\ 2 Astronomical Institute of the Romanian Academy, 5 Cuţitul de Argint, 040557 Bucharest, Romania \\ e-mail: [nedelcu;octavian;petre;paras]@aira.astro.ro \\ 3 Observatoire de Paris, Systemes de Referecnce Temps Espace (SYRTE), CNRS/UMR8630 Paris, France \\ e-mail: jean.souchay@obspm.fr \\ ${ }^{4}$ Universidade Federal do Rio de Janeiro, Observatorio do Valongo, Ladeira Pedro Antonio 43, CEP 20.080 - 090 Rio De Janeiro \\ RJ, Brazil \\ e-mail: massaf@ov.ufrj.br \\ 5 Observatorio Nacional/MCT, R. General Jose Cristino 77, CEP 20921-400 Rio de Janeiro RJ, Brazil \\ e-mail: oat1@on.br
}

Received 24 August 2009 / Accepted 24 September 2009

\section{ABSTRACT}

\begin{abstract}
Aims. We investigate the link between the International Celestial Reference Frame (ICRF) and the dynamical reference frame realized by the ephemerides of the Solar System bodies.

Methods. We propose a procedure that implies a selection of events for asteroids with accurately determined orbits crossing the CCD field containing selected quasars. Using a Bulirsch-Stoer numerical integrator, we constructed 8-years (2010-2018) ephemerides for a set of 836 numbered near-Earth asteroids (NEAs). We searched for close encounters (within a typical field of view of groundbased telescopes) between our selected set of asteroids and quasars with high-accuracy astrometric positions extracted from the Large Quasars Astrometric Catalog (LQAC).

Results. In the designated period (2010-2018), we found a number of 2924, 14 257, and 6972 close approaches (within 10') between asteroids with a minimum solar elongation value of $60^{\circ}$ and quasars from the ICRF-Ext2, the Very Large Baseline Array Calibrator Survey (VLBA-CS), and the Very Large Array (VLA), respectively. This large number of close encounters provides the observational basis needed to investigate the link between the dynamical reference frame and the ICRF.
\end{abstract}

Key words. methods: statistical - astrometry - reference systems - ephemerides - minor planets, asteroids: general

\section{Introduction}

One of the aims of modern astrometry is to establish and maintain links between different reference frames. The link between the International Celestial Reference Frame (ICRF) and Hipparcos Catalog Reference Frame (HCRF) was investigated by direct astrometry of ICRF optical counterparts (Zacharias et al. 1999; da Silva Neto et al. 2000). More recently, using the best available representation of HCRF - the UCAC2 cata$\log$ (Zacharias et al. 2004) - precise optical astrometry of ICRF sources was performed in both southern (Assafin et al. 2005) and northern (Assafin et al. 2007) hemispheres.

Another important connection under study is the one between ICRF and the dynamical reference frame, a frame realized by the ephemerides of the Solar System bodies. For the inner

* Table 2 is also available in electronic form at the CDS via anonymous ftp to cdsarc.u-strasbg. fr $(130.79 .128 .5)$ or via http://cdsweb.u-strasbg.fr/cgi-bin/qcat?J/A+A/509/A27
Solar System this link was directly investigated by VLBI observations of planet-orbiting spacecrafts and angularly nearby quasars (Newhall et al. 1986). In the case of the outer Solar System, simultaneous CCD observations of planets and quasars during apparent close approaches were obtained for Neptune and Uranus (da Silva Neto et al. 2005). In the framework of the forthcoming GAIA space mission, the close approaches of Jupiter with a large sample of quasars from the Véron-Cetty \& Véron (2003) catalog were investigated in the time interval 2005-2015 (Souchay et al. 2007). A substantial number of events observable from both GAIA and Earth with emphasis on the parallax effect was found, allowing a precise tie between the Solar System dynamical reference frame and the extragalactic ICRF.

In this paper we will also focus on the link between the dynamical reference frame and the ICRF. The observational basis needed to investigate this link is represented by the apparent close approaches between Solar System bodies and quasars as objects defining a quasi-inertial reference frame. Thus, we decided to choose the near Earth asteroids (NEAs) to realize 
the dynamical reference frame by means of their ephemerides. Owing to the relative large sample (836 objects) this approach yields more events than the previous works. Additionally, for sufficiently bright asteroids, the relative astrometry in the frame of extragalactic radiosources is much more precise than in the case of the planets, thanks to their star-like appearance. Moreover, the asteroids positions are directly obtained relative to the quasars positions, unlike the planets where this task was achieved using their satellites' relative astrometry as an intermediary step (Souchay et al. 2007).

We have developed and used a numerical integrator to construct 8-years (2010-2018) ephemerides for a set of 836 NEA. We have searched for apparent close encounters (geocentric asteroid - quasar separation within a typical field of view of ground-based telescopes) between our selected set of asteroids and quasars with high-accuracy astrometric positions. Finally, the discussion of results and observational constrains are presented.

\section{Apparent close approaches}

One of the methods for obtaining an accurate tie between different reference frames is the simultaneous observation of an object in both frames. In our case, where the link between dynamical reference frame and ICRF is investigated, this method will require CCD imaging of an asteroid and of an angular nearby quasar. Direct comparison of the asteroid position obtained from a dynamical model of the Solar System with the quasar optical position in an optimally calibrated astrometric field will yield the local, instantaneous orientation of the dynamical reference frame with respect to a quasi-inertial, extragalactic reference frame to a precision higher than that of the usual indirect methods.

To obtain an accurate comparison of the two frames, we have to select asteroids with reliably determined orbits and quasars having high-accuracy astrometric positions.

\subsection{Asteroids}

Near-Earth objects (NEOs) are a population with Earth-crossing orbits generally having the perihelion distances $q \leq 1.3 \mathrm{AU}$ and aphelion distances $Q \geq 0.983$ AU. Among this population there is a subclass of potentially hazardous asteroids (PHA) represented by objects with an absolute magnitude $H \leq 22$ (corresponding to a diameter of $1 \mathrm{~km}$ or larger) having a minimum orbit intersection distance (MOID) of $0.05 \mathrm{AU}$ or less. Dynamical calculations show that the typical lifetime of NEOs is much shorter than the age of the Solar System. They are removed from the inner Solar System in few million years ending their lives by falling onto the Sun (Farinella et al. 1994), by impacting the terrestrial planets (usually Venus or Earth) or by acquiring high-eccentricity, ejection orbits (Gladman et al. 2000). These short lifetimes require stable sources to resupply the NEO. Finding these sources with the associated mechanisms for resupplying the NEOs population is a fundamental problem of today astronomy.

Investigation of the important link between these sources and NEOs requires combined knowledge of their dynamical and physical evolutions and properties. Continuous optical and radar observations are needed for accurate orbit determination and physical characterisation of NEOs.

In our analysis we selected the numbered (observed at two or more oppositions) NEAs from the Asteroid Orbital Elements Database ASTORB (Bowell 2009). This set currently contains
836 asteroids. Our choice was motivated by their orbital elements' accuracy, quantified by the current ephemerides uncertainty (CEU) parameter ( $1 \sigma$ absolute positional uncertainty) for an epoch near the publication date. Thus $91 \%$ of the selected asteroids have $\mathrm{CEU} \leq 0.25^{\prime \prime}$. Moreover the NEA population among all other asteroid populations has shorter periods, making it a reliable set of dynamical reference frame representatives. The orbits are determined by optical and radar observations which represent two good-quality, independent sets of astrometric feed, not available in general for other populations.

To obtain accurate ephemerides of the selected asteroids we used a simplified model of the Solar System along with a numerical integrator. The asteroids' ephemerides are obtained by a simultaneous numerical integration of the equations of motion for the 8 planets (Mercury to Neptune), 4 largest asteroids - (1) Ceres, (2) Pallas, (4) Vesta, and (134340) Pluto, and the asteroid itself (with zero mass). Additional perturbations due to general relativistic effects are only considered for the Sun (Quinn et al. 1991) modeled as a point mass in the isotropic, parametrized post-Newtonian (PPN) metric (Will 1974). Earth and Moon are treated as separate point masses ignoring the figure and the tidal effects. The second-order ordinary differential equations (ODE) of motion are solved using the 12th order (nominal) implicit Bulirsch-Stoer method of Bader \& Deuflhard (1983) with automatic step size control provided by the opensource C library, GNU Scientific Library (GSL). The BulirschStoer method (Bulirsch \& Stoer 1966) is a well known, generalpurpose, self-adapting method of solving ODEs. It was proved to be fast and accurate (Murison 1989), and was applied to a variety of problems associated with the dynamic of few-body system (Charnoz et al. 2001; Tuomi \& Kotiranta 2009; Groussin et al. 2007).

The heliocentric positions and velocities for the planetary and the main 4 perturbing asteroids at the initial epoch of the integrator (JD 2454900.5 - the epoch of the used ASTORB file) were obtained from DE405. The initial conditions for the asteroid (treated as a test particle) were derived from the corresponding heliocentric osculating orbital elements from ASTORB. All masses were taken from DE405. The integration was done in double precision mode and the step size was imposed by the requirement to maintain a relative error of less than $10^{-14}$. To speed up the calculations, the end state of the integrator at a given epoch $\mathrm{T}$ is saved in a temporary cache file and used as an initial state by the subsequent integrations to epochs closer to $\mathrm{T}$ than to the initial epoch of the integrator.

The stability of the integrator was evaluated by performing a backward integration to 1980 followed by a forward integration of 60 years for 3 asteroids: (99942) Apophis, (1862) Apollo, and (433) Eros (Fig. 1). After the forward integration, the positions of the 3 asteroids at the initial epoch were recovered to within $900 \mathrm{~m}$ for Apophis, $80 \mathrm{~m}$ for Apollo, and $20 \mathrm{~m}$ for Eros. The higher values in the case of (99942) Apophis come from multiple, close (within $0.1 \mathrm{AU}$ ), planetary encounters with Venus and Earth in the designated time interval (1980.0-2009.19) keeping the integrator from meeting the $10^{-14}$ error requirement (Giorgini et al. 2008).

The accuracy of our simplified mathematical model was estimated by comparison with JPL's Horizons system (Giorgini et al. 1996) that is using the same algorithms as JPL spacecraft navigation, asteroid radar tracking and planetary ephemeris development. Since we want the comparison to reflect only the differences in the numerical approaches and in the Solar System models, the initial conditions for the 3 asteroids above were obtained from the Horizons system. The results are displayed in 


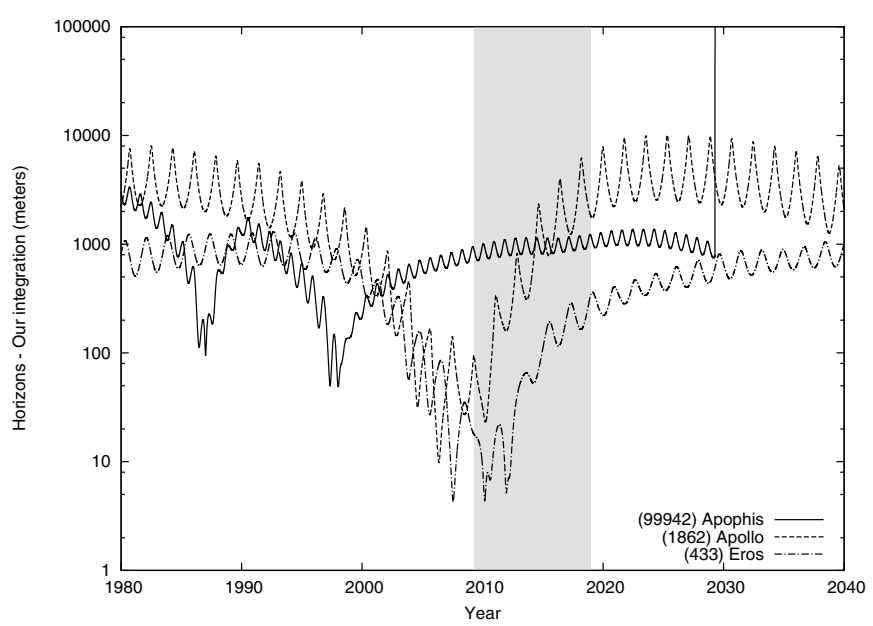

Fig. 1. Differences in heliocentric positions between our numerical integrator and JPL Horizons for (99942) Apophis, (1862) Apollo, and (433) Eros using the same initial conditions.

Fig. 1 and show that a numerical integrator package using a reasonably accurate model of the Solar System can produce planetary positions in almost real-time for a short timespan (typically years) with a precision comparable to JPL models.

\subsection{Quasars}

The Large Quasar Astrometric Survey (LQAC) is a compiled catalog aiming to provide a homogeneous description of the largest 12 quasar catalogs (4 from radio interferometry programs, 8 from optical surveys) with their best position estimates and including physical information in both optical and radio domains (Souchay et al. 2009). With 113666 quasars included, it is currently the largest compiled catalog of confirmed quasars catalogs. Moreover the coordinates of all the objects are given with the best accuracy (in that aim the existing catalogs are ranked according to their astrometric quality).

The ICRF constructed from S and X band VLBI observations is the current realization of the International Celestial Reference System at radio wavelengths. It has 212 defining (setting the ICRF axes) sources with individual positions better than $1 \mathrm{mas}$ that were chosen based on their observing history, the stability, and accuracy of their position estimates. In the original realization ICRF also included positions for 396 sources to make the frame denser (Ma et al. 1998). Later ICRF was extended to include an additional 109 sources (Fey et al. 2004).

The VLBA Calibrator Survey (VCS) catalog included in the LQAC contains 3357 sources (mainly quasars) having similar accuracies to those of ICRF-Ext. 2 catalog (i.e. 1 mas). These positions have been derived from astrometric analyses of the dual frequency VLBA observations.

The Very Large Array (VLA) is the last catalog of quasars with the highly accurate, astrometric positions included in LQAC. The positions were obtained using the VLA interferometer consisting of 27 radio-antennas in a Y-shaped configuration located in New Mexico. Based on the accuracy of source positions (Claussen 2006) 1701 quasars with astrometric precision around 10 mas where included in the LQAC.

The three catalogs, ICRF-Ext.2, VLBA/VCS, and VLA, were designated in the LQAC by the flags $\mathrm{A}, \mathrm{B}$, and $\mathrm{C}$ in the decreasing order of their astrometric accuracy. We estimated the sky density of these 3 catalogs by conventionally defining
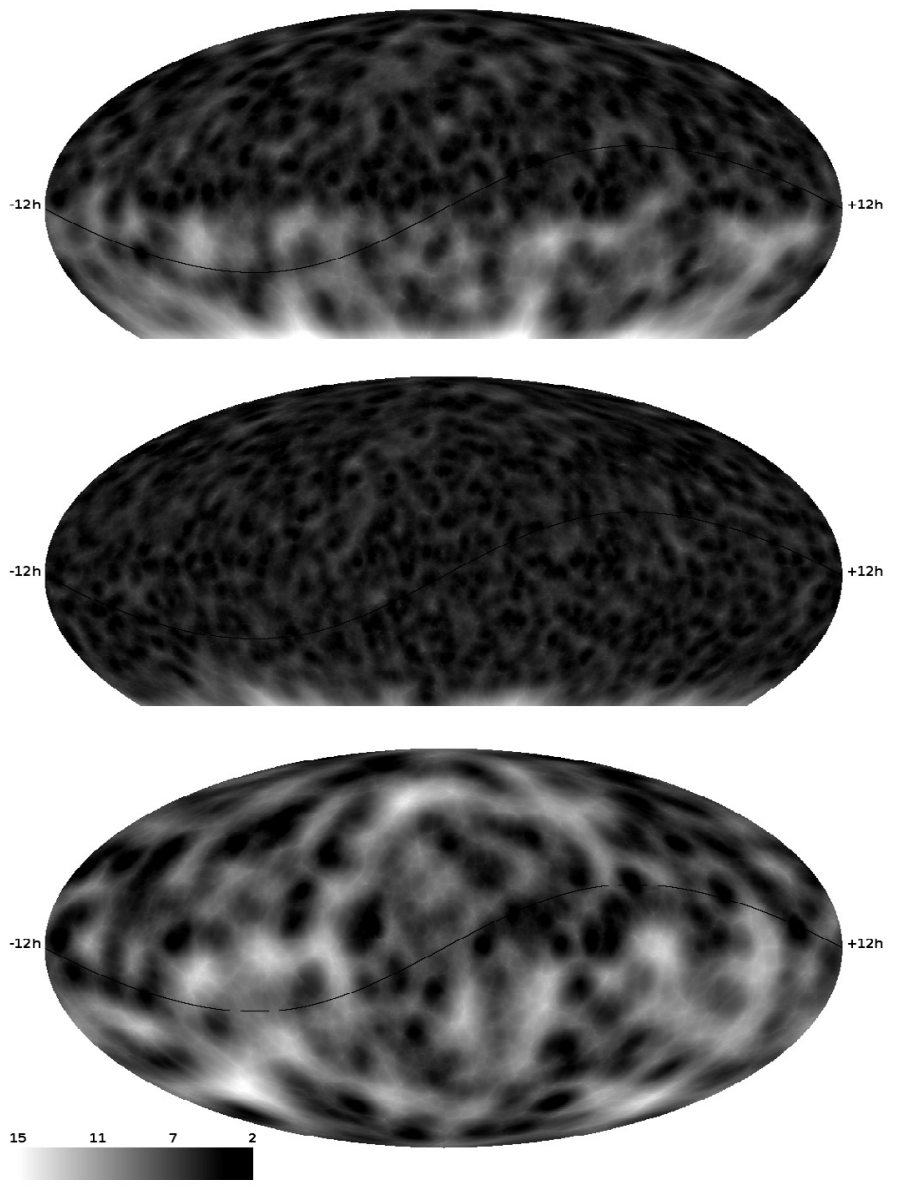

Fig. 2. Sky coverage of quasars subsets A, B, and C (from bottom to top) from LQAC (ICRF-Ext.2, VCS, and VLA, respectively) in J2000 equatorial frame with a Mollweide projection. The ecliptic is represented by the thin black line. The average distance to the nearest 5 sources (in degrees) is color-coded in the image.

a sky-coverage metric as the average distance to the nearest 5 sources. This sky density provides direct information on the number of the quasi-inertial reference points available for differential astrometry in a given region of the sky. It also identifies the low-density area needing future improvement of the celestial reference frame in terms of astrometric quality and density. The strong non-uniformity of the quasar catalogs is evident in Fig. 2. We distinguish a clear dichotomy in the density of quasars between the northern and southern hemispheres. Consequently, this dichotomy affects the regions around the ecliptic where the close approach events are mainly occurring.

With a total of 3530 (uniquely identified) quasars having astrometric positions accurate to the miliarcsecond level, these three catalogs are useful for future investigations of the construction of a denser celestial reference frame at optical and radio wavelengths, and of the connection between the optical, radio, and dynamical reference frames.

\subsection{Events}

The asteroid heliocentric positions and velocities obtained by the numerical integrator are handled by the Naval Observatory Vector Astrometry Subroutines (NOVAS) to obtain the final astrometric, geocentric right ascension and declination. NOVAS is a software library for astrometry-related numerical 
computations based on vector astrometry theories and IAU resolutions (Kaplan et al. 1989; Kaplan \& Bangert 2006).

Since the procedure of finding apparent close approaches between asteroids and quasar requires frequent comparison of their coordinates, we chose to represent the asteroids geocentric right ascension and declination separately using Chebyshev polynomials. The 2010-2018 interval of our numerical integration was divided into contiguous 30 days subintervals. The number of Chebyshev coefficients per interval was kept constant (15) for both geocentric right ascension and declination. Without being too computationally expensive this uniform approach offers a good interpolation precision, even if the asteroids coordinates are changing fast as in the vicinity of oppositions. The maximum position error due to interpolation was checked and found to be less than 0.1 mas in both coordinates.

With asteroids coordinates available now at any given time in the 2010-2018 interval, we can systematically search for apparent close approaches between our selected set of asteroids and the quasars from the LQAC. We followed the method presented by Berthier (1997) to study the occultation of stars by asteroids using an iterative procedure based on the Brent algorithm (Press et al. 1992) to find the minimum asteroid-quasar distance and the time of the closest approach. Since we want these close approaches to form an observational basis for analysis of the link between the dynamical reference frame and the ICRF, we impose a minimum solar elongation value of $60^{\circ}$.

\section{Results and discussion}

In the designated period (2010-2018) we found a number of 2924, 14257, and 6972 close approaches (within 10') between asteroids and quasars from ICRF-Ext2, VLBA-CS, and VLA, respectively. Since these 3 catalogs are partially overlapping, the total number of unique events is 14817 . The events distribution in the above time interval is displayed in Fig. 3. The 1 -year period observable in the events number is a combination of the apparent non-isotropy of the NEA distribution from a terrestrial point of view (NEAs generally having low elongations) and of the sky non-uniformity of the quasar catalogs. Periods with more close approaches correspond to a large number of asteroids crossing sky regions with a higher density of quasars. Accordingly this effect is more pronounced for catalogs with high non-uniformity (ICRF-Ext2, VLA) and less visible in the case of catalogs with more uniform coverage, such as VLBA-CS.

Establishing a link between the ICRF (represented by quasars as fiducial points) and the dynamical reference frame realized by the asteroids ephemeris requires an optimal determination of the differential positions between these moving objects and the quasars. For this reason we have to investigate the possibility of performing differential astrometry in the quasar's field by finding the number of stars that could be used as astrometric standards. Using the $c d s c l i e n t$ package we queried the VizieR service (Ochsenbein et al. 2000) to obtain the number of UCAC2 stars, together with their associated positional errors in a $10^{\prime} \times 10^{\prime}$ field centered on the quasars $\mathrm{J} 2000$ position.

The results are displayed in the Table 1 and show that most of the quasars from ICRF-Ext2, VLBA-CS and VLA catalogs are located in regions with enough UCAC2 stars to allow an accurate astrometric calibration of the instrument field. Moreover, the presence of UCAC2 stars in the quasar vicinity means that the same CCD observations of the event itself (the close approach) could also be used to astrometrically calibrate the telescope field and to derive a field distortion pattern. In a relatively small field

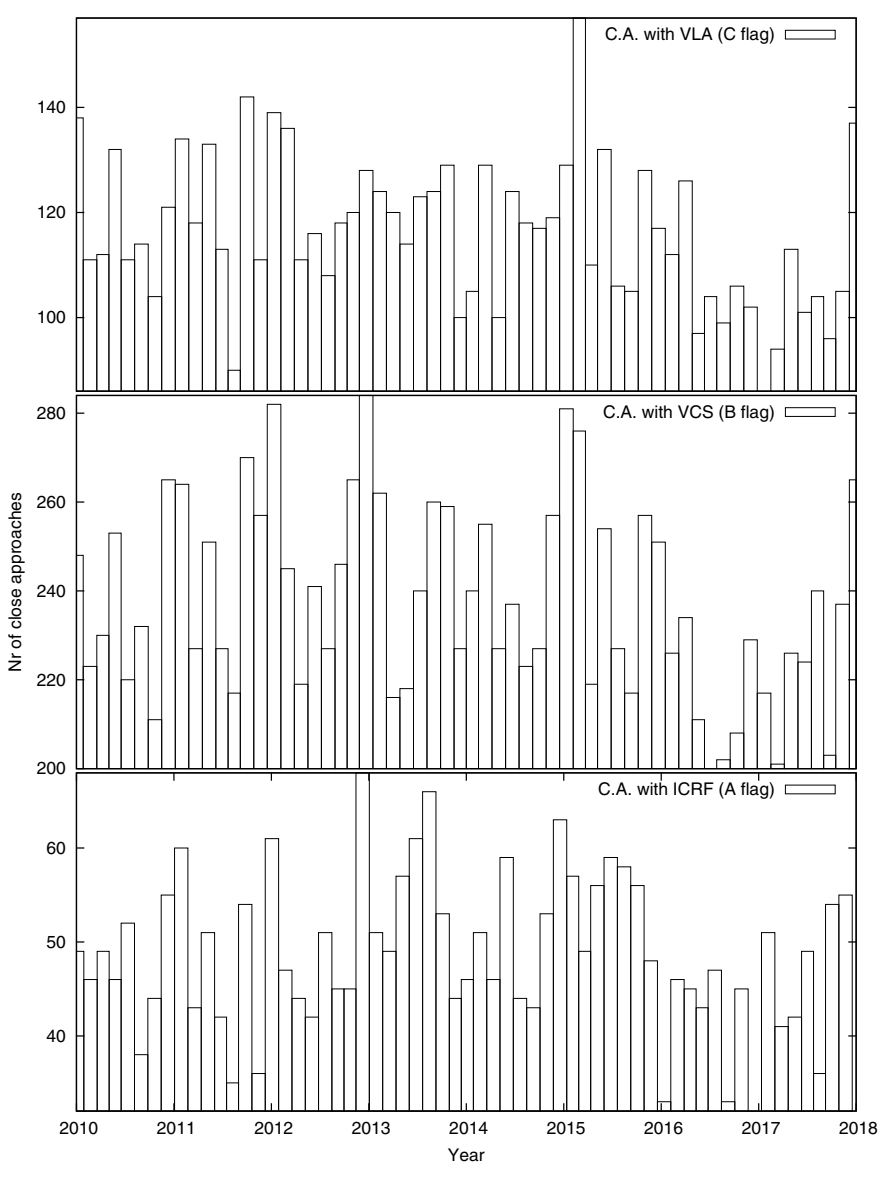

Fig. 3. Distribution of the apparent close approaches between NEA and quasars from ICRF-Ext2, VLBA-CS, and VLA in the 2010-2018 time interval.

of view, the stars from any optical representative of the ICRF (USNOB1.0, UCAC2, etc.) form a locally rigid reference frames free of the systematic positional errors that affects the catalogs on larger scales. The combination of ever increasing precision and density, of the modern catalogs (UCAC3 or the SDSS DR7) make this scheme ever more practical.

By acquiring a large number of frames per field, we obtain the asteroids' position in the ICRF-VLBI frame with an accuracy higher than that of the typical ground-based observations used to compute their orbits where the error budget is dominated by the reference star catalog systematic error (Bowell et al. 2002). To evaluate the procedure of astrometric calibration we used previously acquired images of the quasar J08157+2413 (ICRS coordinates $\alpha=08^{\mathrm{h}} 15^{\mathrm{m}} 45^{\mathrm{s}} \cdot 67, \delta=+24^{\circ} 13^{\prime} 12^{\prime \prime}$. 0 ). A total of 97 images were obtained using the same telescope and observing strategy as presented by Assafin et al. (2007). The detector employed this time was a thermoelectrically cooled Proline CCD camera with an E2V back-illuminated sensor with a $2048 \times$ 2048 pixels array used in the $2 \times 2$ bining mode. In this configuration the instrument field of view was $\sim 12^{\prime} \times 12^{\prime}$. The data reduction followed the standard procedure of bias dark and flat corrections. Precise objects positions were obtained by fitting a general two-dimensional elliptical Gaussian function at the coordinates of a first centroid estimation. A total of 36 UCAC2 stars were found in the field and were used to obtain a standard six-constants full linear plate solution. Using all the 97 frames, the computed mean and standard deviation of plate scale was 


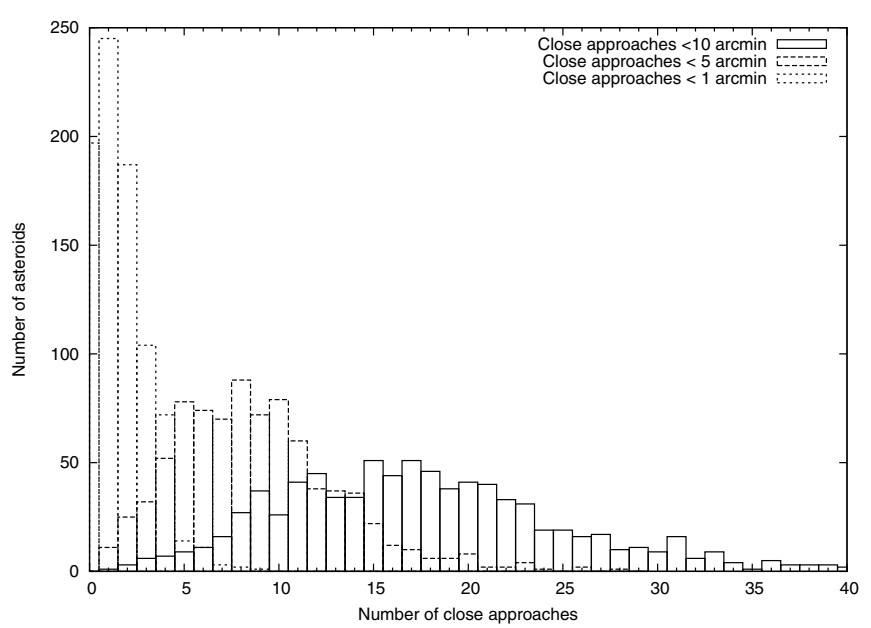

Fig. 4. Histograms of the number of close approaches within $1^{\prime}, 5^{\prime}$, and $10^{\prime}$ respectively.

\section{$\left(0.74657 \pm 4 \times 10^{-5}\right)^{\prime \prime} /$ pixel for $x$ and $\left(0.74660 \pm 7 \times 10^{-5}\right)^{\prime \prime} /$ pixel} for $y$ axes.

From these values we can see that within our field of view (1024 pixels, 12') the maximum error in differential astrometry due to the field calibration amounts to 70 mas. For closer approaches the main source of error will be the centroiding procedure used to obtain $(x, y)$ positions of the asteroid and the quasar. This error depends on the magnitude and the seeing and displays a typical behavior increasing at the faint magnitude end. In our previous work (Assafin et al. 2007), this error was between 50 mas and 100 mas at the detection limit (19 mag) depending on the sky transparency. A general formulation of the astrometric error (in any axis) is $\sigma_{x(y)}=\sigma_{\mathrm{PSF}} / S N R$ (Neuschaefer \& Windhorst 1995) where $\sigma_{\mathrm{PSF}}$ is the Gaussian sigma of the point spread function (PSF) and $S N R$ the peak signal to noise ratio. From the above formula we observe that the measurement error of an object depends on the instrument's focal length, on the detector pixel size, and on the seeing (via $\sigma_{\mathrm{PSF}}$ ) but also on the instrument aperture, quantum efficiency of the detector, and on the exposure time (via $S N R$ ).

Because of their short period orbit, the NEAs from our selected set will have more close approaches with quasars than the outer planets that were previously used in the frame linking. In Fig. 4 we display the distribution of the close approaches events per asteroids. We can see that most of the asteroids have multiple passage in the quasars fields in the 2010-2018 time interval. This allows not only repeated evaluations of the relative orientation of the dynamical reference frame and the ICRF but also frequent, high accuracy measurements of asteroids positions. This set of optical data together with radar data can be used in constraining NEA dynamics and possibly revealing more subtle, non-gravitational effects as the Yarkovsky effect.

Table 2 lists the closest approaches of relatively bright asteroids and quasars that were found between 2010 and 2018. The longer passages and the accesible magnitudes place these events within the capabilities of the small-apperture, narrow field of view telescopes. Additionally, the events where the minimum geocentric asteroid-quasar separation is smaller than the asteroid horizontal parallax could be followed as possible occultations.

In the decade to come, space-based optical astrometry missions such as GAIA will observe a large number of extragalactic objects, many of them quasars, with a projected accuracy that is similar to that of the current radio observations. This makes
Table 1. UCAC2 stars in $10^{\prime} \times 10^{\prime}$ fields around quasars from ICRF-Ext2, VCS, and VLA catalogs.

\begin{tabular}{cccccccc}
\hline \hline & \multicolumn{3}{c}{ UCAC2 Stars } & \multicolumn{2}{c}{$\sigma_{\alpha}$ (mas) } & \multicolumn{2}{c}{$\sigma_{\delta}$ (mas) } \\
& $<4$ & $4-10$ & $>10$ & $<30$ & $>30$ & $<30$ & $>30$ \\
\hline ICRF-Ext2 & 123 & 168 & 425 & 360 & 249 & 404 & 205 \\
VCS & 622 & 761 & 1972 & 1620 & 1190 & 1863 & 947 \\
VLA & 401 & 348 & 951 & 756 & 581 & 848 & 489 \\
\hline
\end{tabular}

The first 3 columns represent the number of quasars fields with the given number of stars from UCAC2 catalog. The next 4 columns denote the number of fields with the average positional errors of the UCAC2 stars $<30$ mas and $>30$ mas.

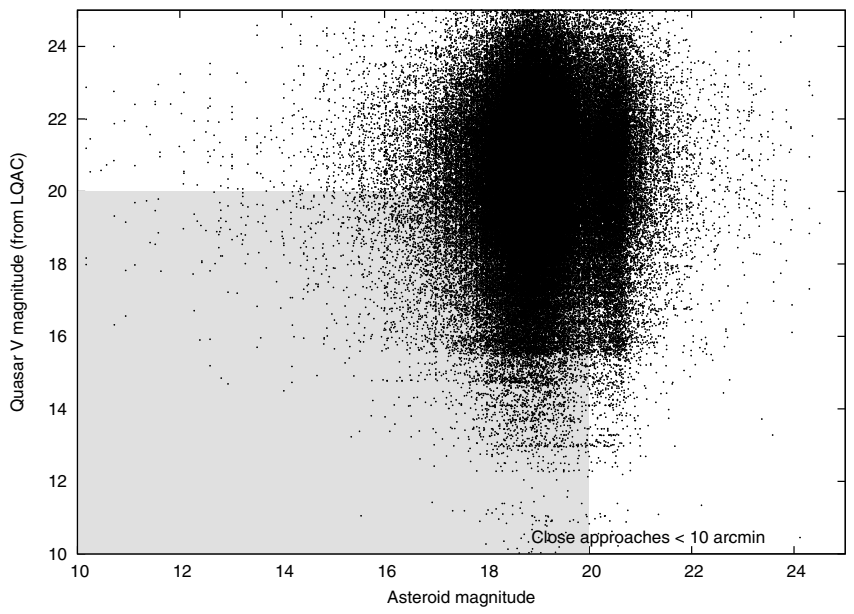

Fig. 5. The magnitude distribution of apparent close approaches between the numbered NEAs and all LQAC quasars. The shaded area represents the location of the events available for observations to typical ground-based observatories.

any close approach between NEA and quasars worthy of interest as long as highly accurate, astrometric positions of the quasars can be obtained in the near future. Meanwhile, the Large Quasar Reference Frame (LQRF) (Andrei et al. 2009) aims to provide an optical representation of the ICRS by homogenizing the astrometry of quasars from different catalogs. With quasar positions placed onto the UCAC2-based reference frame this catalog is already an improvement in terms of astrometric accuracy.

Now with all 111366 quasars from LQAC, we have obtained a very large number of apparent close approaches with NEAs (within $10^{\prime}$ ). Figure 5 shows the relative size for the number of observable events where both objects (quasar and asteroid) are brighter than 20 mag thus making the events relatively accessible for a typical ground-based observatory.

\section{Conclusions}

One of the methods used to obtain an accurate tie between the different reference frames is the simultaneous observation of an object in both frames. To investigate the link between the dynamical reference frame realized by the ephemerides of NEA and the ICRF, we searched for apparent close approaches between a set of numebered asteroids and quasars with high-accuracy astrometric positions.

In the time interval 2010-2018, we found a number of 2924, 14257, and 6972 close approaches (within 10') between asteroids with a minimum solar elongation value of $60^{\circ}$ and quasars from the ICRF-Ext2, the Very Large Baseline Array Calibrator Survey (VLBA-CS), and the Very Large Array 
Table 2. Closest apparent approaches of relatively bright asteroid and quasars in 2010-2018.

\begin{tabular}{|c|c|c|c|c|c|c|c|c|c|c|c|c|c|}
\hline \multirow[b]{2}{*}{ LQAC Flag } & \multicolumn{4}{|c|}{ Quasar } & \multirow[b]{2}{*}{ V } & \multirow[b]{2}{*}{$R$} & \multicolumn{3}{|c|}{ Event } & \multicolumn{2}{|c|}{ Asteroid } & \multicolumn{2}{|r|}{ UCAC2 } \\
\hline & $\alpha\left(^{\circ}\right)$ & $\delta\left(^{\circ}\right)$ & $U$ & $B$ & & & $I$ & (TT) & $\mathrm{Nr}$ & Mag & Dist $(")$ & $\operatorname{SEP}\left({ }^{\circ}\right)$ & Stars \\
\hline $\mathrm{ABC}$ & 14.45368060000 & 30.35244780000 & - & 12.28 & - & 11.26 & 10.15 & $2010 / 1 / 30.27665$ & 85804 & 19.76 & 26.30 & 76.5 & 20 \\
\hline$-B-$ & 224.36129875900 & -35.65276997200 & - & 18.12 & - & 18.40 & 17.36 & $2010 / 2 / 03.92417$ & 163081 & 19.51 & 1.34 & 82.8 & 36 \\
\hline$-\mathrm{BC}$ & 29.73447434100 & 13.11742784440 & 19.02 & 19.29 & 18.66 & 18.83 & 18.60 & $2010 / 2 / 08.77972$ & 9950 & 19.51 & 9.29 & 72.5 & 10 \\
\hline $\mathrm{ABC}$ & 66.19517520000 & 0.60175828600 & 15.66 & 16.15 & 16.05 & 16.42 & 15.38 & $2010 / 3 / 08.18221$ & 143947 & 18.87 & 10.07 & 77.9 & 13 \\
\hline$-\mathrm{BC}$ & 143.80683925500 & 9.25217559443 & 19.83 & 19.75 & 19.82 & 19.34 & 19.09 & $2010 / 5 / 02.55875$ & 159518 & 19.90 & 4.66 & 101.1 & 6 \\
\hline$-B-$ & 3.47554567654 & -4.39785955530 & - & 19.48 & - & 19.17 & 16.73 & $2010 / 5 / 28.85015$ & 177255 & 19.69 & 1.13 & 65.9 & 9 \\
\hline$-B-$ & 269.49510424900 & 5.53000657187 & - & - & - & 18.52 & 17.52 & $2010 / 6 / 05.79201$ & 161998 & 17.21 & 4.57 & 147.9 & 76 \\
\hline $\mathrm{ABC}$ & 158.26544900000 & 41.26839800000 & 19.46 & 18.27 & 18.20 & 19.15 & 19.06 & $2010 / 6 / 16.68266$ & 162980 & 18.61 & 27.43 & 62.7 & 7 \\
\hline -B- & 353.83505035200 & -1.51933102760 & - & 18.67 & 18.78 & 17.97 & 17.25 & $2010 / 6 / 28.94995$ & 19764 & 17.02 & 14.88 & 103.2 & 6 \\
\hline $\mathrm{ABC}$ & 49.96356 & 19.02535860000 & - & 19.92 & 18.81 & 17.96 & 17.38 & $2010 / 8 / 01.15159$ & 4954 & 15.01 & 10.00 & 76.1 & 17 \\
\hline $\mathrm{ABC}$ & 254.53754800000 & 7.69098354000 & - & 19.85 & - & 19.24 & 18.40 & $2010 / 8 / 31.16228$ & 142464 & 19.50 & 8.67 & 94.1 & 28 \\
\hline $\mathrm{ABC}$ & 51.40339310000 & 22.40010150000 & - & 19.96 & 19.11 & 19.00 & 17.94 & $2010 / 10 / 25.58796$ & 88959 & 18.03 & 28.57 & 156.9 & 13 \\
\hline -B- & 318.82681483400 & -14.27871440500 & - & 18.90 & - & 18.12 & 17.82 & $2010 / 12 / 05.65826$ & 164400 & 18.78 & 5.50 & 63.6 & 26 \\
\hline$-B-$ & 194.78525843200 & -23.17740399100 & - & 18.15 & - & 16.14 & 16.36 & $2011 / 2 / 11.39651$ & 3288 & 15.56 & 19.02 & 118.3 & 10 \\
\hline -B- & 256.58540625500 & 12.14994287490 & - & 19.34 & 18.73 & 18.69 & 18.73 & $2011 / 2 / 27.77037$ & 13651 & 18.66 & 3.56 & 85.6 & 32 \\
\hline$-B C$ & 164.91267787600 & 20.95609880280 & - & 17.91 & 18.14 & 18.34 & 17.89 & $2011 / 4 / 08.00906$ & 7480 & 19.93 & 28.30 & 138.4 & 6 \\
\hline$-B-$ & 141.45818503600 & 16.97005716930 & - & 17.45 & 17.49 & 16.83 & 16.80 & $2011 / 5 / 31.54826$ & 141525 & 19.28 & 25.50 & 68.9 & 12 \\
\hline$-B-$ & 183.37370504500 & -24.88692350200 & - & 19.90 & - & 19.50 & 18.85 & $2011 / 5 / 31.60402$ & 172722 & 17.94 & 18.41 & 121.2 & 16 \\
\hline$-B-$ & 351.89993707300 & 15.55265971950 & 18.15 & 18.01 & 17.64 & 17.60 & 17.57 & $2011 / 8 / 12.23487$ & 138524 & 17.20 & 2.94 & 137.0 & 7 \\
\hline $\mathrm{ABC}$ & 337.66782700000 & -39.71446300000 & - & 17.40 & 18.50 & 16.30 & 15.90 & $2011 / 10 / 05.83635$ & 169675 & 18.09 & 13.60 & 125.9 & 13 \\
\hline -B- & 57.79573718700 & -11.88962904100 & - & 19.87 & - & 18.52 & 18.66 & 2011/10/17.63182 & 8035 & 19.41 & 13.42 & 138.6 & 11 \\
\hline -B- & 164.57458681200 & 19.86413071100 & 15.88 & 16.69 & 16.25 & 16.96 & 16.48 & $2011 / 11 / 23.06005$ & 162474 & 19.34 & 5.60 & 82.3 & 8 \\
\hline$-B C$ & 128.06683426600 & 18.53670370550 & - & 17.61 & 17.05 & 15.36 & 14.77 & $2011 / 12 / 01.14132$ & 141018 & 19.77 & 6.16 & 122.6 & 21 \\
\hline -B- & 36.42462453840 & 11.57374025550 & - & 19.09 & 18.00 & 18.34 & 17.60 & $2011 / 12 / 11.87625$ & 162273 & 19.36 & 10.54 & 138.5 & 10 \\
\hline$-B C$ & 325.65375701500 & -4.62875356990 & - & 16.97 & - & 16.33 & 16.34 & $2011 / 12 / 15.94816$ & 172034 & 19.96 & 16.41 & 63.2 & 22 \\
\hline $\mathrm{ABC}$ & 359.88825300000 & 38.84508840000 & - & 19.40 & - & 18.66 & 18.41 & $2011 / 12 / 29.56623$ & 90147 & 18.63 & 21.02 & 98.5 & 20 \\
\hline $\mathrm{ABC}$ & 93.45891330000 & 26.07686660000 & - & 18.50 & 18.00 & 17.14 & 16.52 & $2012 / 1 / 30.33214$ & 25916 & 19.10 & 13.67 & 143.4 & 71 \\
\hline $\mathrm{ABC}$ & 124.56666500000 & 42.37928190000 & - & 18.83 & 18.50 & 18.49 & 17.06 & $2012 / 2 / 19.46045$ & 143651 & 18.20 & 3.38 & 140.9 & 9 \\
\hline$-B C$ & 324.65492134000 & -24.66512953600 & - & 18.73 & 18.60 & 18.68 & 19.54 & $2012 / 4 / 29.95302$ & 2063 & 19.99 & 12.10 & 81.1 & 17 \\
\hline$-\mathrm{BC}$ & 340.45715538600 & 9.89790138350 & - & 18.54 & 19.50 & 18.06 & 17.80 & $2012 / 5 / 06.61321$ & 3752 & 19.56 & 6.98 & 61.8 & 15 \\
\hline -B- & 127.70726680700 & -6.60555639420 & - & 19.63 & - & 18.83 & 19.21 & $2012 / 6 / 02.45217$ & 162186 & 17.12 & 28.22 & 62.9 & 26 \\
\hline$-\mathrm{BC}$ & 318.74305675800 & 28.54922137470 & - & 19.21 & 19.03 & 18.92 & 18.06 & $2012 / 7 / 29.79382$ & 20460 & 18.52 & 28.51 & 132.0 & 55 \\
\hline$-B-$ & 212.29990063500 & -23.26378150800 & - & 15.46 & - & 13.95 & 14.06 & $2012 / 8 / 05.03878$ & 433 & 13.98 & 22.59 & 85.3 & 22 \\
\hline$-B-$ & 104.24658403300 & 3.26484666930 & - & 19.64 & 18.19 & 18.26 & 17.70 & $2012 / 9 / 11.18009$ & 2063 & 17.56 & 15.35 & 65.2 & 82 \\
\hline A- & 98.94378310000 & -75.27133800000 & 15.48 & 16.08 & 15.75 & 15.87 & 15.35 & $2012 / 9 / 14.80671$ & 6455 & 14.78 & 7.42 & 89.0 & 33 \\
\hline $\mathrm{ABC}$ & 294.86107400000 & -15.42862700000 & - & 19.49 & 19.40 & 18.78 & 18.42 & $2012 / 10 / 16.05672$ & 3908 & 17.89 & 1.83 & 91.1 & 59 \\
\hline$-B C$ & 278.04602704800 & -20.66338976900 & - & 17.54 & - & 17.07 & 16.05 & $2012 / 10 / 24.29225$ & 5626 & 18.85 & 17.58 & 66.5 & 219 \\
\hline$-\mathrm{BC}$ & 52.31397865610 & 35.16833078880 & - & 17.86 & 17.47 & 17.50 & 16.77 & $2012 / 10 / 31.88390$ & 99907 & 15.38 & 28.92 & 154.7 & 40 \\
\hline$-\mathrm{BC}$ & 114.24197378100 & 26.08054016960 & - & 18.56 & 18.39 & 18.23 & 17.92 & $2012 / 11 / 07.73283$ & 11284 & 18.67 & 19.51 & 113.7 & 30 \\
\hline$-\mathrm{C}$ & 205.75074800000 & 28.73541600000 & 16.80 & 17.00 & 17.07 & 17.23 & 16.96 & $2012 / 11 / 28.80342$ & 137924 & 18.59 & 4.88 & 62.8 & 13 \\
\hline$-B C$ & 201.83741250000 & 43.44110812500 & 19.78 & 19.63 & 19.66 & 19.18 & 18.91 & 2012/12/11.40715 & 137924 & 18.28 & 26.28 & 84.5 & 6 \\
\hline -B- & 7.26496775845 & 5.15968548032 & - & 19.44 & 18.71 & 18.39 & 17.83 & $2012 / 12 / 15.26079$ & 3908 & 17.37 & 21.27 & 105.2 & 8 \\
\hline -B- & 337.91510926800 & -14.37304762700 & - & 17.94 & - & 17.32 & 16.86 & $2012 / 12 / 22.42420$ & 154715 & 19.93 & 20.92 & 63.6 & 11 \\
\hline$-B C$ & 356.77015419600 & 51.70496570260 & - & 12.70 & 12.43 & 10.49 & 10.64 & $2013 / 2 / 11.02500$ & 6047 & 18.04 & 5.66 & 71.5 & 28 \\
\hline -B- & 177.76334857000 & -17.39995640200 & - & 18.39 & 19.00 & 18.59 & 18.15 & $2013 / 2 / 23.31019$ & 96315 & 16.90 & 15.94 & 145.6 & 8 \\
\hline$-B-$ & 63.36766506830 & -6.25041426110 & - & 18.47 & - & 17.29 & 16.96 & $2013 / 3 / 16.79103$ & 17188 & 18.64 & 20.63 & 66.9 & 18 \\
\hline$-B-$ & 325.19525049500 & -19.39890033200 & - & 19.08 & - & 19.01 & 18.56 & $2013 / 4 / 29.06758$ & 85804 & 18.22 & 5.33 & 77.7 & 14 \\
\hline$-\mathrm{BC}$ & 346.15181835400 & 23.51878082770 & - & 19.61 & 18.98 & 19.10 & 18.55 & $2013 / 6 / 19.96865$ & 24445 & 18.22 & 1.88 & 91.2 & 20 \\
\hline$-\mathrm{BC}$ & 32.80490571260 & 10.85966627750 & - & 16.10 & 15.79 & 15.11 & 14.50 & $2013 / 6 / 28.60267$ & 35107 & 18.87 & 14.46 & 62.6 & 18 \\
\hline$-B-$ & 235.14396074600 & -39.10493467400 & - & 16.66 & 17.57 & 16.36 & 15.07 & 2013/9/12.69286 & 7839 & 19.68 & 5.18 & 73.1 & 93 \\
\hline $\mathrm{ABC}$ & 294.31757200000 & -39.96709800000 & - & 17.66 & - & 17.61 & 17.34 & $2013 / 9 / 20.30159$ & 86829 & 19.60 & 4.11 & 110.9 & 40 \\
\hline$-B C$ & 112.08586791300 & 21.88510843600 & - & 19.00 & 18.70 & 18.25 & 18.31 & $2013 / 10 / 06.09328$ & 18109 & 19.76 & 10.91 & 82.4 & 36 \\
\hline$-B-$ & 289.43674716900 & -19.35878042200 & - & 15.68 & 15.59 & 15.73 & 14.77 & $2013 / 11 / 03.28598$ & 86039 & 18.74 & 17.79 & 67.5 & 146 \\
\hline$-B-$ & 137.30065629800 & 8.59474976433 & 18.63 & 18.06 & 17.57 & 17.75 & 17.29 & $2013 / 11 / 08.51223$ & 6611 & 17.83 & 23.36 & 88.9 & 24 \\
\hline $\mathrm{ABC}$ & 92.42062300000 & -15.71129800000 & - & - & 17.00 & - & - & $2013 / 11 / 18.82271$ & 138883 & 19.14 & 17.30 & 128.5 & 31 \\
\hline -B- & 326.87968940700 & -36.03091192400 & - & 19.29 & 18.20 & 17.93 & 18.20 & 2013/12/04.91843 & 3361 & 18.06 & 17.02 & 65.8 & 11 \\
\hline $\mathrm{ABC}$ & 341.57596700000 & -12.11424400000 & 16.02 & 16.63 & 16.45 & 16.26 & 15.94 & 2013/12/16.25649 & 5189 & 19.75 & 11.07 & 74.2 & 15 \\
\hline -B- & 358.53823296600 & -0.32998770810 & 18.41 & 18.11 & 19.00 & 17.92 & 17.70 & 2013/12/16.89255 & 163696 & 16.81 & 24.07 & 93.6 & 10 \\
\hline $\mathrm{ABC}$ & 149.58196500000 & 47.41884510000 & 18.54 & 18.69 & 18.00 & 18.48 & 18.18 & $2013 / 12 / 26.25601$ & 53435 & 15.71 & 12.53 & 130.6 & 8 \\
\hline -B- & 3.76038353741 & -18.21413417100 & - & 19.69 & - & 19.11 & 18.77 & $2014 / 1 / 06.97225$ & 10563 & 19.42 & 25.86 & 70.6 & 6 \\
\hline$-B-$ & 239.95684296200 & -5.52293551650 & - & 18.72 & - & 18.27 & 17.51 & $2014 / 2 / 03.57772$ & 25916 & 17.35 & 17.27 & 76.0 & 18 \\
\hline$-B-$ & 100.02998454100 & -12.88765785200 & - & 16.58 & - & 15.27 & 15.37 & $2014 / 3 / 06.11441$ & 138947 & 18.65 & 12.83 & 111.5 & 81 \\
\hline
\end{tabular}


Table 2. continued.

\begin{tabular}{|c|c|c|c|c|c|c|c|c|c|c|c|c|c|}
\hline \multirow[b]{2}{*}{ LQAC Flag } & \multirow[b]{2}{*}{$\alpha\left({ }^{\circ}\right)$} & \multicolumn{3}{|l|}{ Quasar } & \multirow[b]{2}{*}{ V } & \multirow[b]{2}{*}{$R$} & \multirow{2}{*}{\multicolumn{2}{|c|}{ Event }} & \multicolumn{3}{|c|}{ 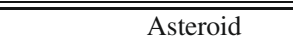 } & \multicolumn{2}{|r|}{$\overline{\mathrm{UCAC} 2}$} \\
\hline & & $\delta\left(^{\circ}\right)$ & $U$ & $B$ & & & & & $\mathrm{Nr}$ & Mag & $\operatorname{Dist}(")$ & $\operatorname{SEP}\left({ }^{\circ}\right)$ & Stars \\
\hline$-\mathrm{BC}$ & 315.25691655400 & -29.55773223000 & - & 18.50 & - & 18.20 & 18.08 & $2014 / 4 / 15.52298$ & 39572 & 18.66 & 9.70 & 76.4 & 22 \\
\hline$-\mathrm{B}-$ & 315.97184003100 & -9.56157044720 & - & 14.55 & - & 12.87 & 13.34 & $2014 / 4 / 20.81518$ & 66391 & 18.24 & 10.78 & 75.0 & 13 \\
\hline$-\mathrm{B}-$ & 233.01071534100 & -27.27719966100 & - & 19.16 & - & 18.52 & 18.12 & $2014 / 6 / 02.09057$ & 163335 & 19.23 & 18.04 & 164.0 & 38 \\
\hline$-\mathrm{B}-$ & 190.15559277500 & -11.35688821900 & - & 19.41 & - & 18.82 & 18.80 & $2014 / 6 / 14.49840$ & 37336 & 19.77 & 11.35 & 110.5 & 9 \\
\hline $\mathrm{ABC}$ & 166.78622600000 & -44.81878300000 & - & 17.70 & 18.20 & 17.29 & 16.81 & $2014 / 7 / 15.67735$ & 17511 & 16.88 & 29.94 & 81.5 & 56 \\
\hline$-\mathrm{BC}$ & 44.71052633920 & 5.68556629154 & - & 19.79 & 18.71 & 19.37 & - & $2014 / 9 / 08.57467$ & 4401 & 17.83 & 3.08 & 121.1 & 13 \\
\hline$-\mathrm{BC}$ & 313.28901671100 & 22.80041294990 & - & 19.35 & - & 17.88 & 17.89 & $2014 / 9 / 09.64123$ & 138847 & 18.54 & 26.76 & 136.2 & 74 \\
\hline $\mathrm{ABC}$ & 256.89339700000 & 1.81269423000 & - & - & 18.90 & - & - & $2014 / 9 / 30.47234$ & 68548 & 18.32 & 5.55 & 70.6 & 27 \\
\hline $\mathrm{ABC}$ & 75.30337450000 & -1.98729340000 & - & 19.06 & 18.40 & 19.06 & 18.12 & $2014 / 10 / 18.24621$ & 154300 & 18.99 & 9.03 & 126.4 & 20 \\
\hline $\mathrm{ABC}$ & 66.17601550000 & -37.93910700000 & 17.46 & 18.15 & 18.08 & 17.75 & 17.95 & $2014 / 11 / 24.83669$ & 177016 & 19.86 & 18.18 & 121.2 & 16 \\
\hline$-B-$ & 1.32472419738 & -16.80129968800 & - & 18.10 & - & 17.96 & 17.44 & $2014 / 11 / 25.84672$ & 136897 & 19.42 & 20.84 & 110.2 & 8 \\
\hline $\mathrm{ABC}$ & 209.27530900000 & -17.73386200000 & - & 19.24 & - & 18.71 & 18.57 & $2014 / 12 / 30.43227$ & 137108 & 19.45 & 16.11 & 65.1 & 11 \\
\hline$-\mathrm{BC}$ & 115.77127832100 & 17.24011471370 & - & 19.16 & - & 17.16 & 17.92 & $2015 / 1 / 06.10557$ & 85804 & 15.14 & 22.07 & 169.8 & 43 \\
\hline $\mathrm{ABC}$ & 169.61232300000 & -46.57083400000 & 16.86 & 17.30 & 17.00 & 16.52 & 16.00 & $2015 / 1 / 14.92734$ & 4957 & 18.70 & 10.17 & 96.8 & 59 \\
\hline$-\mathrm{BC}$ & 245.99271626400 & 7.69181967523 & 18.30 & 19.57 & 18.83 & 17.89 & 17.78 & $2015 / 2 / 13.30792$ & 7822 & 19.64 & 15.44 & 82.5 & 23 \\
\hline $\mathrm{ABC}$ & 111.46100000000 & -0.91570677000 & - & 18.13 & - & 16.06 & 15.93 & $2015 / 2 / 14.03439$ & 5587 & 18.87 & 6.70 & 142.0 & 81 \\
\hline$-B-$ & 350.93596467900 & -16.21447855500 & - & 18.52 & - & 17.95 & 17.54 & $2015 / 5 / 16.92272$ & 7335 & 18.08 & 20.25 & 70.7 & 8 \\
\hline$-B-$ & 42.06178386210 & 4.57801731957 & - & 9.66 & 10.17 & 8.13 & 8.43 & $2015 / 9 / 14.43244$ & 154807 & 18.16 & 11.70 & 129.1 & 12 \\
\hline$-B-$ & 122.61009983200 & 10.17805432740 & - & 17.73 & 18.07 & 17.34 & 17.00 & $2015 / 10 / 10.60825$ & 66146 & 15.76 & 21.10 & 74.4 & 25 \\
\hline$-B-$ & 296.31634495200 & 9.88321214146 & - & 19.54 & - & - & - & 2015/11/06.41606 & 99907 & 18.60 & 19.56 & 78.9 & 111 \\
\hline$-B-$ & 313.96333061800 & -12.57895475200 & - & 19.59 & - & 18.28 & 18.75 & $2015 / 11 / 26.66597$ & 31345 & 19.95 & 23.19 & 69.0 & 23 \\
\hline$-B-$ & 172.14808848600 & 21.04371209670 & - & 18.92 & 19.26 & 18.65 & 19.15 & $2016 / 3 / 13.79377$ & 8013 & 17.71 & 8.62 & 161.5 & 10 \\
\hline$-\mathrm{B}-$ & 177.56917783400 & 43.53497377770 & - & 19.74 & - & 19.00 & - & $2016 / 5 / 27.99510$ & 154652 & 19.69 & 8.96 & 90.4 & 6 \\
\hline$-B-$ & 213.83680802100 & -9.93286920220 & - & 17.50 & - & - & - & $2016 / 8 / 01.55023$ & 8013 & 18.68 & & .5 & 12 \\
\hline$-B-$ & 207.65059987700 & -16.58042146600 & - & 15.23 & - & 12.88 & 13.89 & $2016 / 8 / 09.54536$ & 7092 & 18.64 & 12.30 & 74.5 & 12 \\
\hline$-\mathrm{B}-$ & 235.14396074600 & -39.10493467400 & - & 16.66 & 17.57 & 16.36 & 15.07 & $2016 / 8 / 28.65062$ & 162463 & 19.81 & 4.22 & 86.7 & 93 \\
\hline$-\mathrm{BC}$ & 46.17234352420 & 33.81209181670 & - & 18.13 & 18.70 & 18.45 & 18.83 & $2016 / 9 / 04.15924$ & 5836 & 14.87 & 12.81 & 107.8 & 19 \\
\hline $\mathrm{ABC}$ & 66.19517520000 & 0.60175828600 & 15.66 & 16.15 & 16.05 & 16.42 & 15.38 & $2016 / 9 / 09.45707$ & 52750 & 17.36 & 21.28 & 101.7 & 13 \\
\hline$-B-$ & 97.47929582590 & -5.08335316380 & - & 19.53 & - & 18.85 & 18.58 & $2016 / 9 / 27.42641$ & 52750 & 17.04 & 11.59 & 86.5 & 103 \\
\hline$-\mathrm{B}-$ & 308.22631251000 & -21.42105554400 & - & 19.53 & - & 18.33 & 17.68 & $2016 / 11 / 06.85710$ & 163249 & 18.66 & 18.15 & 80.6 & 37 \\
\hline$A B-$ & 61.95179580000 & -12.19351700000 & 14.93 & 15.53 & 15.35 & 14.43 & 13.91 & $2016 / 11 / 23.05857$ & 96590 & 13.23 & 10.31 & 147.3 & 13 \\
\hline$-B C$ & 356.77015419600 & 51.70496570260 & - & 12.70 & 12.43 & 10.49 & 10.64 & $2016 / 12 / 08.95877$ & 152563 & 18.08 & 4.43 & 114.3 & 28 \\
\hline $\mathrm{ABC}$ & 111.32003200000 & 14.42048520000 & - & 18.59 & 17.75 & 17.53 & 17.02 & $2016 / 12 / 28.54474$ & 12923 & 19.70 & 7.25 & 164.3 & 40 \\
\hline$-B-$ & 119.21124569500 & -15.70151000500 & - & 19.13 & - & 18.32 & 17.87 & $2017 / 2 / 19.79410$ & 1036 & 14.73 & 5.86 & 137.1 & 102 \\
\hline$-B-$ & 301.32205480700 & -18.36758969100 & - & 18.51 & - & 18.02 & 17.87 & $2017 / 3 / 25.08205$ & 68950 & 18.53 & 10.07 & 64.8 & 42 \\
\hline $\mathrm{ABC}$ & 350.88314100000 & -3.28472880000 & - & 19.34 & 18.60 & 18.38 & 17.98 & $2017 / 5 / 21.05002$ & 177614 & 19.30 & 18.15 & 69.6 & 12 \\
\hline$-B-$ & 341.96933774900 & -12.62214477800 & - & 18.38 & - & 17.93 & 17.63 & $2017 / 5 / 27.81605$ & 5660 & 18.67 & 26.34 & 87.9 & 8 \\
\hline$-B-$ & 337.91510926800 & -14.37304762700 & - & 17.94 & - & 17.32 & 16.86 & $2017 / 6 / 21.47208$ & 96631 & 19.03 & 22.48 & 115.7 & 11 \\
\hline $\mathrm{ABC}$ & 343.28070500000 & 19.70961910000 & - & 17.19 & 16.66 & 16.26 & 15.79 & $2017 / 9 / 08.99474$ & 90075 & 15.69 & 15.14 & 154.6 & 16 \\
\hline$-\mathrm{BC}$ & 128.47452241500 & 42.40051402490 & 17.82 & 17.89 & 18.72 & 16.83 & 16.50 & $2017 / 9 / 23.24605$ & 137078 & 18.82 & 28.23 & 62.8 & 16 \\
\hline$-\mathrm{B}-$ & 9.44226239522 & 36.98635632480 & - & 19.49 & 18.21 & 17.51 & 16.93 & $2017 / 10 / 08.61844$ & 31221 & 19.73 & 28.73 & 148.7 & 35 \\
\hline$-\mathrm{B}-$ & 31.81503808100 & -38.95085383600 & - & 18.99 & - & 16.29 & 16.39 & $2017 / 11 / 22.77538$ & 2329 & 16.36 & 11.29 & 115.9 & 11 \\
\hline $\mathrm{ABC}$ & 323.54295700000 & -1.88812180000 & - & 19.60 & 18.73 & 18.66 & 17.73 & 2017/12/09.79157 & 68950 & 17.95 & 2.16 & 68.1 & 11 \\
\hline$-B-$ & 7.26496775845 & 5.15968548032 & - & 19.44 & 18.71 & 18.39 & 17.83 & $2018 / 1 / 02.58488$ & 162998 & 19.09 & 22.36 & 86.8 & 8 \\
\hline
\end{tabular}

The columns are in order: the LQAC flags of quasars subsets (A, B, C for quasars with highly accurate, radio positions) quasar J2000 equatorial coordinates, $U, B, V, R$, and $I$ magnitudes, the Terestrial Time of the close approach event in the year/month/day format, NEA number, apparent visual magnitude, the minimum geocentric asteroid - quasar separation ( $\operatorname{arcsec})$, the solar elongation angle and number of UCAC2 stars in a $10^{\prime} \times 10^{\prime}$ field centered at the quasar position that could be used for astrometry.

(VLA),respectively. This large number of close encounters provides the observational basis needed to investigate the link between the dynamical reference frame and the ICRF.

Acknowledgements. Valuable comments from an anonymous referee are greatly acknowledged. The work of D.A.N , O.B , P.P., and P.P. was supported by the European Space Agency in the framework of the PECS program.

\section{References}

Andrei, A. H., Souchay, J., Zacharias, N., et al. 2009, A\&A, 505, 385

Assafin, M., Monken Gomes, P. T., da Silva Neto, D. N., et al. 2005, AJ, 129 2907
Assafin, M., Nedelcu, D. A., Badescu, O., et al. 2007, A\&A, 476, 989

Bader, G., \& Deuflhard, P. 1983, Numer. Math., 41, 373

Berthier, J. 1997, Journal des Astronomes Francais, 55, 47

Bowell, E. 2009, VizieR Online Data Catalog, 1, 2001

Bowell, E., Virtanen, J., Muinonen, K., \& Boattini, A. 2002, Asteroids III, 27

Bulirsch, R., \& Stoer, J. 1966, Numer. Math., 8, 1

Charnoz, S., Thébault, P., \& Brahic, A. 2001, A\&A, 373, 683

Claussen, M. 2006, VLA calibrator manual

da Silva Neto, D. N., Andrei, A. H., Martins, R. V., \& Assafin, M. 2000, AJ, 119 , 1470

da Silva Neto, D. N., Assafin, M., Andrei, A. H., \& Vieira Martins, R. 2005, in

The Three-Dimensional Universe with Gaia, ed. C. Turon, K. S. O'Flaherty,

\& M. A. C. Perryman (ESA Special Publication), 576, 285

Farinella, P., Froeschle, C., Froeschle, C., et al. 1994, Nature, 371, 315 
Fey, A. L., Ma, C., Arias, E. F., et al. 2004, AJ, 127, 3587

Giorgini, J. D., Benner, L. A. M., Ostro, S. J., Nolan, M. C., \& Busch, M. W. 2008, Icarus, 193,

Giorgini, J. D., Yeomans, D. K., Chamberlin, A. B., et al. 1996, BAAS, 28, 1158 Gladman, B., Michel, P., \& Froeschlé, C. 2000, Icarus, 146, 176

Groussin, O., Hahn, G., Lamy, P. L., Gonczi, R., \& Valsecchi, G. B. 2007, MNRAS, 376, 1399

Kaplan, G. H., \& Bangert, J. A. 2006, Nomenclature, Precession and New Models in Fundamental Astronomy, 26th meeting of the IAU, Joint Discussion 16, 22-23 August, Prague, Czech Republic, JD16, \#3, 16

Kaplan, G. H., Hughes, J. A., Seidelmann, P. K., Smith, C. A., \& Yallop, B. D. 1989, AJ, 97, 1197

Ma, C., Arias, E. F., Eubanks, T. M., et al. 1998, AJ, 116, 516

Murison, M. A. 1989, AJ, 97, 1496

Neuschaefer, L. W., \& Windhorst, R. A. 1995, ApJS, 96, 371
Newhall, X. X., Preston, R. A., \& Esposito, P. B. 1986, in Astrometric Techniques, ed. H. K. Eichhorn, \& R. J. Leacock, IAU Symp., 109, 789 Ochsenbein, F., Bauer, P., \& Marcout, J. 2000, A\&AS, 143, 23

Press, W. H., Teukolsky, S. A., Vetterling, W. T., \& Flannery, B. P. 1992, Numerical recipes in $\mathrm{C}$. The art of scientific computing (Cambridge: University Press)

Quinn, T. R., Tremaine, S., \& Duncan, M. 1991, AJ, 101, 2287

Souchay, J., Le Poncin-Lafitte, C., \& Andrei, A. H. 2007, A\&A, 471, 335

Souchay, J., Andrei, A. H., Barache, C., et al. 2009, A\&A, 494, 799

Tuomi, M., \& Kotiranta, S. 2009, A\&A, 496, L13

Véron-Cetty, M.-P., \& Véron, P. 2003, A\&A, 412, 399

Will, C. M. 1974, ApJ, 191, 521

Zacharias, N., Zacharias, M. I., Hall, D. M., et al. 1999, AJ, 118, 2511

Zacharias, N., Urban, S. E., Zacharias, M. I., et al. 2004, AJ, 127, 3043 